\title{
Parentalidades gays y lesbianas: los cambios de relato
}

\author{
Micaela Cynthia Libson \\ Doctora en Ciencias Sociales (Universidad de Buenos Aires) \\ Becaria CONICET en la Universidad de Buenos Aires \\ micaelalibson@hotmail.com
}

\begin{abstract}
Resumen En este artículo analizo los relatos que narran gays y lesbianas sobre las parentalidades y las familias. Describo el campo de la intimidad a partir de tres relatos típicos: el de la imposibilidad, el de la oportunidad y el de la elección. El estudio se apoya en una investigación empírica y cualitativa realizada en los meses de septiembre de 2008 a enero de 2009 en Buenos Aires, Argentina. Realicé 22 entrevistas a varones gays y mujeres lesbianas con hijos/as o proyecto de tenerlos/as.
\end{abstract}

Palabras-clave: parentalidad, gays, lesbianas, intimidad, heteronormatividad.

\section{Introducción}

$\mathrm{E}$ N este artículo analizo los Relatos que narran gays y lesbianas sobre las parentalidades y las familias. Planteo en el desarrollo una diversidad de historias. Por un lado, relatos que hablan de un pasado lejano en el que las posibilidades de generar progenie para gays y/o para lesbianas les eran ajenas. Por el otro, historias presentes en las que esas posibilidades se manifiestan de manera más certera. Tales cambios se vinculan con transformaciones en las vidas de gays y de lesbianas en el terreno de lo íntimo, producto de la ideología individualista de la modernidad tardía (Giddens, 1995; Beck, 1996, 1997; Castel, 1996; Sennett, 2000). Como se plantea en la literatura angloparlante (Blumer, 1969; Plummer, 2003; Giddens, 2004), el campo de la intimidad conjuga aspectos de las vidas personales que poseen relación directa con el sexo, las sexualidades y las familias.

El estudio que presento se apoya en una investigación empírica y cualitativa realizada en los meses de septiembre a diciembre de 2008 y enero de 2009 en Buenos Aires, Argentina. Realicé 22 entrevistas a varones gays y mujeres lesbianas con hijos/as o proyecto de tenerlos/ as. El criterio para establecer el grupo muestral se determinó por la saturación teórica de los datos (Glaser y Strauss, 1967). Las entrevistadas y los entrevistados fueron reclutados mediante agrupaciones lesbianas, gays, bisexuales, transexuales, travestis e intersex (desde ahora LGTTBI) y a través del método de bola de nieve. Para realizar las entrevistas, se concretaba con el contacto vía mail o telefónicamente, y se acordaba el día, hora y lugar para efectuarla. La mayoría de las entrevistas, fueron hechas en bares y duraron entre 40 minutos y una hora.

Para abordar el trabajo, el artículo se divide en tres secciones. En la primera, se recorren visiones de la sociología familiar, analizadas luego a la luz de la categoría de heteronormatividad. Se aborda tal concepto desde los feminismos, en especial desde el llamado feminismo lesbiano (Rich, 1976; Wittig, 1978, 1987), para contextualizar y justificar la comprensión de facetas familiares que escapan a los modelos teóricos y 
empíricos heteronormativos. En la segunda sección, se describen las transformaciones sucedidas en el campo de la intimidad de gays y de lesbianas. Se analizan tales cambios a partir de los relatos tipificados en lo que Weeks, Heaphy y Donovan (2001: 160-168) describen como: el relato de la imposibilidad, el relato de la oportunidad y el relato de la elección. Cada tipo expresa una forma particular de posicionarse, en tanto gays y lesbianas, respecto del tener y criar sus propios hijos/as.

\section{Heteronormatividad y feminismos: el silenciamiento y la visibilización de la diversidad familiar}

La familia no constituye un fenómeno dado, unívoco, homogéneo, sino que es un fenómeno complejo que hace hablar de ella en plural. Los abordajes legos y científicos sobre ella implican una variedad, incluso contradictoria, de fenómenos muy diversos. Las múltiples construcciones del concepto "familias" se articulan en torno a diferentes saberes, cada uno más o menos hegemónico en su tiempo. Disciplinas como la antropología, la sociología, la demografía, la psicología y la psiquiatría, el derecho, la medicina, la biología, entre otras, reflexionan sobre dinámicas familiares sentando los límites que permitieran cada abordaje. Si se acota al campo de la literatura sociológica y demográfica, varias perspectivas, en especial del mundo angloparlante, parten del modelo de Parsons (1978) como el tipo familiar ideal del equilibrio social. Visiones sustentadas en las teorías sobre la transición demográfica, tanto la clásica (Norestein, 1953) como la segunda transición (Lesthaeghe y Van de Kaa, 1986), las teorías socioeconómicas del intercambio social (Becker, 1987) y del calendario matrimonial (Oppenheimer, 1988) plasman estas consideraciones a partir de erigir a la familia nuclear, biológica, biparental, monogámica y heterosexual como el ideal social de la complementariedad de los sexos.

Los preceptos de las visiones posmodernas, no tan recientes como suele imaginarse, comparten muchas reflexiones con el feminismo (Barrón López, 2008). Si bien se presenta la diferencia como valor epistemológico, son igualmente discernibles ciertos desvelamiento de la mirada funcionalista al poner en el centro del debate la crisis, el desorden (Roudinesco, 2005) y la novedad como discurso mediante los lemas que rezan por las nuevas formas familiares. Uno de los caminos para comprender la tendencia a la nuclearización heterosexual de lo familiar ${ }^{1}$, y que compone una de las principales líneas de las concepciones sobre parentesco, familia y parentalidad dentro del mundo occidental, es la heteronormatividad (Pichardo Galán, 2009). Tal noción es introducida por la poeta y ensayista norteamericana Rich (1976), quien trabaja sobre la institucionalización forzosa de la heterosexualidad en términos de "heterosexualidad obligatoria" (Rich, 1976: 2). Reflexiones similares son desarrolladas por la filósofa francesa Wittig (1978, 1987), para quien el concepto de heterosexualidad puede pensarse en términos contractualistas. Wittig propone la imagen del contrato heterosexual como una metáfora paralela a la sostenida por los pensadores ingleses y franceses del Siglo de las Luces. En esta operación metafórica el pacto se instituye como "una forma ideológica amorfa que no se puede asir en su realidad, salvo en sus efectos, y cuya existencia reside en el espíritu de las gentes de un modo que afecta su vida por completo" (Wittig, 1987: 5), un contrato que relega, que se funde en la naturaleza y en la desigualdad de varones y mujeres, heterosexuales y no heterosexuales. En esa línea, Wittig habla del carácter opresivo del "pensamiento heterocentrado" (Wittig, 1978: 6), en el intento por universalizar la producción de conceptos a partir de la negación de las prácticas concretas.

En concordancia con el pensamiento de Wittig, Pateman explica la historia del contrato social colocándole como precedente el contrato sexual. En esta perspectiva, el contrato originario es un pacto que plantea dos caras. Por un lado, un proceso de sujeción de las mujeres respecto de los varones, a través del contrato sexual. Por el otro, un proceso de libertad de los varones en un orden patriarcal, mediante el contrato social (Pateman, 1995). Dentro del feminismo queer, Kosofsky-Sedgwcik (1998) señala que la heteronormatividad instala un juego de binarismos, tales como masculino/femenino, activo/pasivo, que se articulan en términos de relaciones naturales. Para la autora, el dispositivo de la heterosexualidad reduce la diversidad de lo social a una oposición entre dos, donde lo masculino y lo femenino se constituyen como la base obligatoria de la sociedad.

También en esta línea de análisis, los conceptos de intercambio de mujeres y tabú del incesto esconden para la antropóloga Rubin dos premisas: por un lado, la heterosexualidad como factor incuestionable, por el otro, una teoría de opresión sexual (Rubin, 2000). Como sugiere la autora, "el tabú del incesto presupone un tabú anterior, menos articulado, contra la homosexualidad" (Rubin, 2000). En tal sentido, los sistemas de parentesco no sólo estimulan la heterosexualidad, haciéndola implícita, sino que a su vez exigen formas específicas de vínculos 
sexuales: por una parte, dejan de lado las relaciones no heterosexuales; por la otra, generan una escala de legitimación cultural, social, legal, económica y política sobre qué relaciones heterosexuales son aceptadas y cuáles no (Rubin, 1989).

Como planteé antes, la reflexión que introduce Rich (1976), de pensar la heterosexualidad en términos compulsivos y obligatorios, permiten analizar los modos en que tantos gays como lesbianas vivencian (o han vivenciado) la parentalidad y las familias. En el terreno de lo familiar, la heteronormatividad se hace tangible en prácticas e instituciones, presentadas como las únicas posibles, como la familia biparental nuclear sobre la cual se prescriben dos imperativos: por un lado, la alianza heterosexual sancionada legalmente, bajo un esquema de co-residencia y con un marco de vinculación adulta de pareja parental monógama; por el otro, la descendencia biológica producto de una relación sexual. En la sección siguiente describo la variedad de relatos que narran gays y lesbianas sobre su vinculación con las parentalidades y las familias.

\section{Relatos de la intimidad}

Un modo para indagar los cambios (si es que se está en presencia de los mismos) de las vidas personales de los participantes de este estudio es examinar las historias narradas en la arena de la intimidad (Plummer, 2003). Como sugerí líneas atrás, defino la intimidad como la otra cara de la privacidad (Giddens, 1995), esto es, como un conjunto de expectativas que constituyen un lazo estrecho entre el proyecto reflexivo del yo, y que implica un equilibrio entre los componentes autónomos del sujeto (el mundo privado) y la participación de sentimientos y experiencias.

¿Se pueden situar cambios en las historias íntimas de los gays y las lesbianas que protagonizan este trabajo? Y si es así, ¿de qué nos hablan estos cambios? El relato de Serena, ${ }^{2}$ una de las entrevistadas, es ilustrativo en tal sentido, pues narra su experiencia de lo que para ella significaron los primeros momentos en los que comenzó a reflexionar sobre sus posibilidades personales de ser madre.

No era tan fácil. No conocíamos lesbianas que lo estuvieran haciendo. Participábamos de "Lesbianas a la vista" [organización socio-política de lesbianas], pero no estaban en ese proceso ninguna de nuestras compañeras. Nos ayudaron a buscar y a conseguir y demás, pero no es que estaba instalado. Es más, dentro de las lesbianas o desde las feministas en general ocurría lo contrario, si la maternidad era un imperativo, una no debía embarcarse.

(Serena, mujer lesbiana con hijos).

La historia de Serena da cuenta de lo que Weeks, Heaphy y Donovan (2001) ubican dentro del mundo gay y lesbiano, como el relato de la imposibilidad. Esto es, un relato típico de una generación de varones gays y mujeres lesbianas de finales de los años setentas y principios de los ochentas (en este caso, de Gran Bretaña, pero que se puede atribuir también a otros contextos y/o épocas) que afirman en sus experiencias lo impensable que personas gays y lesbianas tuvieran hijos/as o proyectos de parentalidad. Como señalan los autores, "muchas mujeres y, particularmente, aún más varones nunca han imaginado que podrían convertirse en padres" (Weeks, Heaphy y Donovan, 2001: 161-162). El relato de Serena, si bien es posterior a los años ochenta, se ubica dentro de un contexto argentino diferente al de los Estados Unidos o Gran Bretaña de esa misma época. Mientras que en esos países se plasmaba, como describo luego, el auge del baby boom lesbiano, en la situación local recién comenzaba a darse inicio a una visibilización periodística ${ }^{3}$ destinada a mostrar la cuestión homosexual en la Argentina ${ }^{4}$.

$\mathrm{Si}$ nos retrotraemos un poco en la historia, vemos que en Estados Unidos y Gran Bretaña las investigaciones sobre la temática de parentalidades gays y lesbianas surgieron en los años setenta. A diferencia de la Argentina, tales estudios no fueron el producto de una politización por parte del activismo LGTTBI de ambas regiones, sino que fue la visibilización de experiencias de parejas y parentalidades gays y lesbianas lo que comenzó a ser foco de interés en distintos campos disciplinarios. En cierto sentido, el relato de Serena permite mostrar las distancias existentes entre aquel saber especializado y emergente en los países angloparlantes y las redes de conocimiento compartidas que en el contexto local aún no era acogido ni decodificado (Meccia, 2006).

En el siguiente relato, el de Lorena, una mujer lesbiana de treinta y pocos años, se puede ver cómo se percibe la parentalidad como indisociable de la heterosexualidad.

2. Los nombres de las personas entrevistadas han sido cambiados para mantener la confidencialidad y el anonimato.

3. En abril de 1984, la revista argentina Siete días publica como nota de tapa "Los riesgos de ser homosexual en la Argentina", con una foto del primer presidente de la Comunidad Homosexual Argentina (CHA) Carlos Jáuregui abrazado junto a otro activista de la CHA. Esta fue la primera vez que una persona gay salía en un medio masivo de comunicación. Para mayor información puede consultarse la página de la Comunidad Homosexual Argentina. Disponible en: http://www.cha.org.ar/simple.php?menu=10\&cat=53.

4. Ello corresponde a la década del setenta y principios del ochenta, contexto en el cual el movimiento se incluía en la lucha por el cese de la represión física (Meccia, 2006). 
Entrevistadora: ¿En algún momento vos pensabas que ibas a ser mamá?

Lorena: Sí, siempre quise ser mamá.

Entrevistadora: ¿Y cambió este sentimiento cuando empezaste a sentir que te gustaban las chicas?

Lorena: Bueno mi hija llegó siendo yo muy joven... es decir yo me casé siendo muy joven.

Entrevistadora: ¿Vos te casaste?

Lorena: Sí, yo me casé. Bueno y vino mi hija, después de que ella ya cumpliera un año... un año y pico, me empezaron a caer las fichas de un montón de cosas, con respecto a la vida que estaba llevando. En realidad bueno, o sea, de algún modo el tema también de mi elección sexual fue toda una cuestión.

Entrevistadora: ¿Estabas casada? ${ }^{5}$

Lorena: Estaba casada pero yo sabía que me gustaban las mujeres desde que tenía quince años...Y me casé, porque quería tener un hijo.

(Lorena, mujer lesbiana con hijos.)

La historia de Lorena da cuenta del supuesto extendido de que determinadas prácticas familiares son legítimas y otras no, o no lo son tanto. Como vimos en la primera sección, la heteronormatividad asocia mandatos de heterosexualidad obligatoria para la constitución de las parentalidades. Ello puede observare en el relato de Lorena; esto es, cómo el dispositivo de heterosexualidad opera en sus experiencias y proyectos parentales, incluyéndose en los marcos en los que ese proyecto era factible. Su historia muestra una experiencia desdoblada en la que lo emocional se confronta con lo normativo, la heterosexualidad compulsiva en términos de Rich (1976), al incorporar una norma externa que se rechaza de manera interna.

El relato permite evidenciar tal incorporación: "yo me casé, porque quería tener un hijo" mediante el complejo del amor romántico (Giddens, 2004). Sobre dicha noción, realizo un desarrollo más acabado en el análisis del tercer tipo de relato (el de la elección), pero es importante resaltar aquí cómo tal complejo da cuenta de la asociación tríadica entre amor, maternidad y matrimonio.

Puede observarse otro tipo de relato, caracterizado en la historia de Mauricio, que se describe a continuación.

Me casé con la madre de mis hijos. Me casé hace bastante tiempo. Digamos, estuvimos dos años sin tener chicos. Estuvimos muy bien, hasta que, bueno, nuestro matrimonio entró en crisis. Primero, a mí me empezaron a pasar cosas con hombres, yo pensé que era una cosa pasajera, que bueno, digamos, una negación personal a todo ese tema. La fantasía de que si la gente se enteraba, qué iban a decir, eh...y después que tenía dos chicos. Entonces, digamos, mi vida llegó a una encrucijada. Yo estaba viviendo una vida que no quería vivir. Yo me empecé a dar cuenta de todo esto cuando empecé prácticamente una crisis a los veintisiete años, veintiocho años, yendo a los treinta exploté. Y dije, digamos, tengo dos caminos: o hacer una doble vida y ser un viejo choto infeliz de setenta años, viviendo cagando a mi ex mujer. $\mathrm{O}$ decir, esto se terminó, tengo un hombre al lado mío. Decir, bueno, quiero vivir la vida que yo quiero vivir, y no la que los demás me impusieron. Lo cual me costó un montón, pero creo que se puede, y vale la pena apostar. Y, después de resolver todo el tema de los chicos, que vos no te separás de tus chicos, seguís siendo el mismo papá.

(Mauricio, varón gay con hijos.)

La historia de Mauricio expresa lo que Weeks, Heaphy y Donovan (2001) denominan como el relato de la oportunidad. Esto es, un relato vinculado a un marco concreto de apertura sobre posibilidades antes no problematizadas como el hecho de que gays sean padres y las lesbianas madres. En términos más concretos, y como señalan los autores, ello se ejemplifica en los casos en que "muchas personas no heterosexuales, que tuvieron sus relaciones o matrimonios heterosexuales, hicieron su coming out, y luego reclamaron el derecho de continuar su relación parental" (Weeksk, Heaphy y Donovan, 2001: 164). Tanto en Estados Unidos como en Gran Bretaña ello tuvo mucho auge durante los años setenta frente alto grado de visibilización que tomó las experiencias de mujeres lesbianas con hijos/as conocido como el baby boom lesbiano. Esto es, el surgimiento de una generación de mujeres que comienzan a vivir abiertamente su vida como lesbianas, como mujeres que aman a otras mujeres, y que no están obligadas a casarse con un varón para tener hijos o hijas (Chauncey, 2004).

En el caso de las y los entrevistados, el relato de Mauricio permite mostrar aquel punto de inflexión. Por un lado, porque quiebra la pauta de la heterosexualidad como obligatoria, para la constitución de lazos amorosos: "decir, bueno, quiero vivir la vida que yo quiero vivir, y no la que los demás me impusieron". Por otro lado, porque inaugura la concepción de que gays y lesbianas sean padres y madres, y que la sexualidad y la procreación pueden separarse íntegramente (Giddens, 2004). Este último punto, encuentra una historia aún más pasada con la emergencia de la medicina anticonceptiva (Knibiehler, 2000) y que halla su punto culminante

5. En el momento de las entrevistas el matrimonio entre personas del mismo sexo aún no estaba legislado ni sancionado. Por lo tanto, "estar casada" significa tener un vínculo legal con una persona de distinto sexo. 
con los tratamientos de reproducción asistida (Knibiehler, 2000; Giddens, 2004; Tubert, 2004)

A diferencia del relato de la imposibilidad y del relato de la oportunidad, la mayoría de quienes han sido entrevistados para este estudio han narrado otro tipo de historia. El relato de la elección representa, en términos de Weeks, Donovan y Heaphy, "el cambio más radical en la concepción tradicional de familia" (Weeks, Donovan y Heaphy, 2001: 165). Veamos los distintos testimonios de quienes protagonizan este estudio.

Entrevistadora: ¿En algún momento de tu vida vos quisiste ser papá?

José: No, surgió más que nada ahora porque encontré la persona adecuada. Y fue una decisión de los dos.

(José, varón gay sin hijos.)

Tampoco quiero sonar a vieja, pero como que fuimos una pareja muy constituida y fue como algo más formal por decirlo de alguna manera y fue como algo más... aceptado.

(Carolina, mujer lesbiana con hijos.)

Las historias de José y Carolina permiten dar cuenta de dos puntos que, incluso, pueden interrelacionarse. Por un lado, la presencia de repertorios de configuraciones parentales heterosexuales como los idearios del amor romántico (Giddens, 2004). Relatos como el de José: "surgió más que nada ahora porque encontré a la persona adecuada", evidencian el quiebre entre el amor y la sexualidad, ruptura que era propia de la complementariedad entre el varón y la mujer. En los relatos de ambos se observa la subjetivación de estos valores para la constitución de lazos pues es el amor el que se coloca como el elemento sustancial del ámbito relacional.

Por otro lado, los relatos de José y Carolina también dan cuenta de otro elemento, el de la elección, propio de dos procesos emergentes en la modernidad ya trabajados desde la sociología familiar: la individuación familiar y su consecuente privatización y la desacralización familiar. Autores como Flaquer (1999), Meil (1999), Beck y Beck-Gernsheim (2001), Beck-Gernsheim (2003), Giddens (2004), entre otros, han dado cuenta de cómo el estudio de la configuración familiar ha transitado el paso de una "sociedad de familias hacia otra sociedad de individuos" (Requena y Díez de Revenga, 1993: 250). El sentido cada vez más individualizado de lo social ha impactado en el ámbito familiar, proveyendo un campo en el que las opciones y el establecimiento de relaciones puras constituyen parámetros que otorgan al sujeto un marco de "seguridad ontológica" (Giddens, 1995: 52). Del mismo modo, la secularización de la vida colectiva ha abierto un campo institucional más flexi- ble, en relación a épocas pasadas, en el que la desacralización familiar produjo que las configuraciones familiares, y las mismas estructuras de parentesco, se encuentren más alejadas de los controles religiosos, sociales y culturales.

Los procesos recién enumerados han sido ubicados por la literatura sociológica bajo el signo de la desinstitucionalización de lo familiar (Meil, 1999). Esto es:

La pérdida progresiva de la identidad monolítica de la familia y el debilitamiento de sus aspectos más normativos para convertirse en un espacio interactivo de alto contenido afectivo que escapa en gran medida a controles institucionales y donde cabe el conflicto abierto (público) y legítimo, posibilitando nuevos itinerarios y formas de convivencia a partir de las rupturas de vínculos que protagonizan los sujetos (Barrón López, 2002: 32).

La desinstitucionalización de lo familiar conlleva intrínsecamente el elemento electivo dentro del ámbito de las familias, central en el tipo que Weeks, Heaphy y Donovan (2001) llaman del relato de la elección. La noción de elección es introducida por Weston (2003) en lo concerniente a las familias lésbicas y gays, aunque dicho concepto se aborda en estudios sociológicos anteriores, como los que tratan de la finalización de la pareja conyugal, familias monoparentales o familias ensambladas, entre otras (Gittins, 1985). Pero Weston introduce en su rótulo de "familias elegidas" varios elementos que resultan novedosos. En primer término, toma lo biológico y la elección como una oposición ideológica, como dos principios definitorios que orientan la organización de las relaciones. Como lo plantea la autora:

En la frase 'las familias que elegimos', la apropiación representada por el "elegimos" subraya el papel de cada persona en la creación de las familias lesbi-gays, del mismo modo que la ausencia de apropiación en el término "familia biológica" refuerza el sentido de la consanguinidad como un factor inmutable sobre el que la individualidad ejerce poco control (Weston, 2003: 72).

En segundo término, permite pensar de manera conjunta parentalidad y homosexualidad. Las familias de elección (gay y lésbica) cuestionan esa creencia en dos niveles: la biología y la heterosexualidad. Si bien el concepto de "elección" pareciera solo poner en cuestión al paradigma biologicista, lo singular de la propuesta de Weston es que ello también pone en cuestión al heterosexismo.

Detrás de la noción de elección se esconden, entonces, varios presupuestos. Su crítica no se orienta a 
lo biológico o lo heterosexual per se. No cuestiona el concebir un hijo a la manera biológica (esto es, mediante una unión heterosexual, o fruto de la reproducción asistida con donante o alquiler de vientre). Tampoco cuestiona la heterosexualidad en cuanto tal. Pues, y sí aquí tomo lo que sostiene Butler "las prácticas heterosexuales no son lo mismo que las normas heterosexuales" (Butler, 2006: 282). Su objeto de reflexión es el peso social escondido detrás de la biología y la heterosexualidad que las transforma en normas obligatorias para la constitución de familias. La ligazón que establece entre biología y heterosexualidad no es arbitraria. Es el efecto performativo que poseen al instalarse como matriz de obligatoriedad. Si ambas conforman la norma, lo que las somete a crítica es la elección y la homosexualidad, como otras formas de instituir arreglos familiares. En tal sentido, para quienes protagonizan este estudio, si bien toman como cuestión su experiencia homosexual (y ello puede notarse en el relato de la entrevistada Celeste), marcan una ruptura con los otros tipos de relatos: la posibilidad de generar progenie dentro de una pareja gay o lesbiana.

Entrevistadora: ¿Vos cuándo empezaste a sentir el deseo de ser mamá?

Carolina: Desde que estoy con mi mujer.

(Carolina, mujer lesbiana con hijos.)

Yo pensaba que podía estar con una mina, podía boludear, estaba todo bien, pero que en algún momento iba a estar con un tipo para formar una familia. Pero, cuando empiezo con mi mujer actual, la relación empieza a ser duradera y yo me empiezo a hacer la cabeza y a querer tener hijos con ella.

(Celeste, mujer lesbiana con hijos.)

La mayoría de quienes he entrevistado, han narrado experiencias vinculadas al relato de la elección. Pero se observan variaciones en las historias si se toman en cuenta la variable generacional y cómo han tenido o planean tener sus hijos o hijas. En muchos casos, los relatos responden a historias pasadas, con lo cual si bien se hallaron historias de la imposibilidad, ello no debe interpretarse como experiencias desvinculadas a la parentalidad. Al tomar la primera variable que es la generacional, vemos que quienes se encuentran en el rango de los 40 años o más, relataron experiencias vinculadas con la historia de la imposibilidad y, en menor medida, con la de la oportunidad. La categoría de los 30 a 39 años, engloba a quienes narraron historias de la elección, luego de la oportunidad y, en menor medida, de la imposibilidad. Por último, las personas menores de
30 años relataron experiencias vinculadas a la historia de la elección y, en menor medida, a la oportunidad.

La segunda variable es el modo en que las personas tuvieron o planean tener sus hijos. En este eje observamos que quienes han tenido hijos como producto de uniones heterosexuales anteriores, relataron experiencias vinculadas con la historia de la oportunidad y, en menor medida, con la de la imposibilidad. Quienes buscan tener sus hijos, como se ve en la sección siguiente, a través de un proyecto de co-parentalidad, tecnologías de procreación asistida o adopción, narraron historias de elección.

Las transformaciones producidas en los relatos de gays y lesbianas describen escenarios que se vinculan a distintos contextos generacionales como a situaciones parentales heterogéneas. Y ello se vincula con procesos individuales y personales, y también con procesos coyunturales e históricos. En la Argentina, la reivindicación de demandas familiriastas no solo comprendió los debates en torno a la regulación estatal de las parejas del mismo sexo. Paralelamente, en el año 2000 surgieron agrupaciones de madres lesbianas y padres gays, como Madres Lesbianas Feministas Autónomas, que tuvo sus primeras congregaciones en la organización y asociación civil feminista La Casa del Encuentro, destinadas a ofrecer talleres sobre madres lesbianas desde un relato experiencial y con contenido político. Por su parte, emergieron grupos de reflexión para madres lesbianas y padres gays en la Comunidad Homosexual Argentina y en Puerta Abierta ${ }^{6}$ coordinados por especialistas del campo psi. También en el año 2000, surgieron los grupos Familias Homoparentales Argentinas (FHOA), posteriormente denominada Familias Homoparentales Integradas Argentinas (FHOIAR), Lesmadres y Mis Mamás Rosario. En junio de 2010 se produjo el primer encuentro nacional de familias de parentalidades gays y lesbianas en la Ciudad de Rosario, Santa Fe, convocado por Mis Mamás Rosario en el contexto del debate sobre la Ley de Matrimonio Igualitario. El encuentro logró reunir a más de 68 familias, con el objetivo de lograr puntos comunes para el pedido de mayor reconocimiento jurídico de sus contextos familiares.

La mayoría de estas agrupaciones tuvo como cara visible a las lesbianas más que a los gays. Tales mujeres visibilizaron la existencia de familias co-maternales que reclamaban ante situaciones de desprotección jurídica, en especial para el caso de las madres no gestantes y de sus hijos en relación con ellas.

La toma de conocimiento público de la temática sobre parentalidades gays y lesbianas en la Argentina fue el producto de una estrategia de politización desde el activismo LGTTBI local. Las movilizaciones LGTTBI lograron conquistas jurídicas únicas en la Argentina, a comparación del resto de Latinoamérica. 
La Argentina fue el primer país latinoamericano en sancionar, primero, la ley de Unión Civil en la Ciudad Autónoma de Buenos Aires (diciembre de 2002) y, posteriormente, la Ley de Matrimonio Igualitario (julio de 2010). Asimismo, en la Argentina tales temáticas son aún incipientes e, incluso, la mayoría de las investigaciones han sido producidas en concordancia con activismo local. La razón de ello ha sido la siguiente: otorgar un sustento científico a una estrategia política definida.

\section{Recapitulación y consideraciones finales}

En este artículo analicé las transformaciones producidas en los relatos de gays y lesbianas, con respecto a las configuraciones familiares, en un contexto de heterosexualidad obligatoria. Para ello, estructuré el artículo en dos secciones.

En la primera sección, "Heteronormatividad y feminismos: el silenciamiento y la visibilización de la diversidad familiar", rastreé en los estudios sociológicos sobre familia, la relación entre familia y heterosexualidad, y retomé las consideraciones hechas sobre el concepto de heteronormatividad desde los feminismos, en especial desde el feminismo lesbiano. El desarrollo de la noción de heteronormatividad, como sistema socio-ideológico de estigma y exclusión, permite entender por qué incluso evidenciándose que las dinámicas familiares de parentalidades gays y lesbianas son existentes, igualmente se plantean relaciones de tensión. Esto es, prácticas familiares que, a la vez que subvierten elementos de la heteronormativa familiar, también los reproducen.

\section{Referencias}

BARRÓN LÓPEZ, Sara. Transiciones familiares: monoparentalidad femenina por ruptura conyugal. Tesis ( Doctorado en Sociología) - Universidad del País Vasco/EHU, País Vasco, 2002.

BARRÓN LÓPEZ, Sara. Investigación empírica y teoría feminista en los estudios familiares en el mundo anglosajón: una síntesis extramuros. Empiria. Revista de metodología de ciencias sociales, n 15. Enero-Junio, p. 75-98, 2008.

BECK, Ulrich. Teoría de la modernización reflexiva. In: BERIAN, Josetxo (Comp.), Las consecuencias perversas de la modernidad. Modernidad, contingencia y riesgo. Barcelona: Anthropos, 1996. p. 223-266.

BECK, Ulrich. Hijos de la libertad. México: Fondo de Cultura Económica, 1997.
En la segunda sección, "Relatos de intimidad", mediante un análisis comprensivo de las historias de quienes protagonizan este estudio describí tres relatos. El relato de la imposibilidad, el de la oportunidad y el de la elección. Como se vio en el desarrollo de la sección, dichas construcciones han sido tomadas y adaptadas del trabajo de Weeks, Heaphy y Donovan (2001), para otorgar un marco explicativo a los hallazgos empíricos.

Vimos que el relato de la imposibilidad responde a un contexto específico, de fines de los setenta y principios de los ochenta en la Argentina, en que los gays y lesbianas consideraban inimaginable un escenario de parentalidad. Por su parte, mostré cómo el relato de la oportunidad muestra nuevas realidades, la de gays y lesbianas con hijos (de uniones heterosexuales previas), que permite transformar los esquemas anteriores, en lo que atañe a los repertorios familiares. Por último, presenté el relato de la elección, como aquel que reflejaba la entrada a proyectos parentales diversos en el que se incluyen la bi-parentalidad de gays y de lesbianas. Ahora bien, y para no caer en conclusiones apresuradas, planteo estos datos en términos conjeturales; esto es, como posibles hipótesis para el estudio de futuros desarrollos.

El análisis de los tres relatos me permitió evidenciar una historia dentro del campo de la intimidad que ha sufrido cambios. Las trayectorias biográficas de quienes entrevisté dan cuenta de tales transformaciones como momentos de quiebre dentro del mundo discursivo y vivencial de gays y de lesbianas. Ello otorga un marco explicativo y comprensivo de la incidencia que han tenido esas rupturas para analizar los distintos modos en que gays y lesbianas han tenido o buscan generar descendencia.
BECK, Ulrich y BECK-GERNSHEIM, Elisabeth. El normal caos del amor. Las nuevas formas de la relación amorosa. Buenos Aires: Paidós, 2001.

BECK-GERNSHEIM, Elisabeth. La reinvención de la familia. En busca de nuevas formas de convivencia. Buenos Aires: Paidós, 2003.

BECKER, Gary. Tratado sobre la familia. Madrid: Alianza, 1987.

BLUMER, Herbert. Symbolic Interactionism. Nueva Jersey: Prentice Hall, 1969.

BUTLER, Judith. Deshacer el género. Barcelona: Paidós, 2006.

CASTEL, Robert. La metamorfosis de la cuestión social. Una crónica del salariado. Buenos Aires: Paidós, 1996. 
CHAUNCEY, George. Why Marriage? The history shaping today's debate over gay equality. New York: Basic Books, 2004.

FLAQUER, Luis. La estrella menguante del padre. Barcelona: Ariel, 1999.

GIDDENS, Anthony. Modernidad e identidad del yo. Barcelona: Península, 1995.

GIDDENS, Anthony. La transformación de la intimidad. Sexualidad, amor y erotismo en las sociedades modernas. Madrid: Cátedra, 2004.

GITTINS, Diana. The Family in Question. Basingstoke: Macmillan Press, 1985.

GLASER, Barney y STRAUSS, Anselm. The discovery of Grounded Theory: Strategies of Qualitative Research. Chicago: Aldine, 1967.

KNIBIEHLER, Yvonne. Historia de las madres y de la maternidad en Occidente. Buenos Aires: Nueva Visión, 2000.

KOSOFSKY-SEDGWICK, Eve. Epistemología del armario. Barcelona: Ediciones de la Tempestad, 1998.

LESTHAEGHE, Ron y VAN DE KAA, Dirk J. Bevolking: Krimp and Groei. Deventer: Van Loghum Slaterus, 1986.

MECCIA, Ernesto. La cuestión gay. Un enfoque sociológico. Buenos Aires: Gran Aldea, 2006.

MEIL, Gerardo. La postmodernización de la familia española. Madrid: Acento, 1999.

NORESTEIN, Frank. Economic Problems of Population Change. In: Proceedings of the Eighth International Conference of Agricultural Economists. London: Oxford University Press, p 13-31, 1953.

OPPENHEIMER, Valerie. A Theory of Marriage Timing. American Journal of Sociology, 94. p. 563-591, 1998.

PATEMAN, Carole. El contrato sexual. Barcelona: Anthropos, [1988] 1995.

PARSONS, Talcott. La estructura social de la familia. In: FROMM, Erich, et al. La familia. Barcelona: Península, 1978. p. 31-65.

PICHARDO GALÁN, José Ignacio. Entender la diversidad familiar. Relaciones homosexuales y nuevos modelos de familia. Barcelona: Bellaterra, 2009.

PLUMMER, Ken. La cuadratura de la ciudadanía íntima.
Algunas propuestas preliminares. In: OSBORNE, Raquel y GUASCH, Oscar (Comps.). Sociología de la sexualidad. Madrid: Siglo XXI, 2003. p. 25-48.

REQUENA Y DÍEZ DE REVENGA, Miguel. Formas de familia en la España Contemporánea. In: GARRIDO, Luis y GIL CALVO, Enrique (Eds.). Estrategias familiares, Madrid: Alianza Universidad, 1993. p. 249-270.

RICH, Adrienne. Heterosexualidad obligatoria y existencia lesbiana. Revista Brujas, n. 10, 11, 12. Año 4. Asociación de Trabajo y Estudio de la Mujer, p. 1-28, 1976.

ROUDINESCO, Elisabeth. La familia en desorden. Buenos Aires: Fondo de Cultura Económica, 2005.

RUBIN, Gayle. El tráfico de mujeres: notas sobre la 'economía política' del sexo. In: LAMAS, Marta (comp.). El género. La construcción cultural de la diferencia sexual. México: PUEG, [1975] 2000. p. 35-96.

RUBIN, Gayle. Reflexionando sobre el sexo: notas para una teoría radical de la sexualidad. In: VANCE, Carole (Comp.). Placer y peligro. Explorando la sexualidad femenina. Madrid: Revolución, 1989. p. 113-190.

SENNETT, Richard. La corrosión del carácter. Las consecuencias personales del trabajo en el nuevo capitalismo. Barcelona: Anagrama, 2000.

TUBERT, Silvia. La maternidad en el discurso de las nuevas tecnologías reproductivas. In: DE LA CONCHA, Ángeles y OSBORNE, Raquel (Coords.) Las mujeres y los niños primero. Discursos de la maternidad. Barcelona: Icaria, 2004. p. 111-137.

WESTON, Kath. Las familias que elegimos. Lesbianas, gays y parentesco. Barcelona: Bellaterra, [1997], 2003.

WEEKS, Jeffrey; HEAPHY, Brian; DONOVAN, Catherine. Same Sex Intimacies. Families of choice and other life experiments. New York: Routledge, 2001.

WITTIG, Monique. El pensamiento heterocentrado. (Disponible en: http://www.hartza.com/QUEER.html), p. 1-10, 1978.

WITTIG, Monique. A propósito del contrato social. (Disponible en: http://www.hartza.com/QUEER.html), p. 1-8, 1987. 


\section{Gays and Lesbians' Parenthood: changing the stories}

\section{Abstract}

Based on 22 in-depth interviews with gays and lesbians with children, this paper describes the experiences of gays as fathers and lesbians as mothers. This paper analyses gays' and lesbians' stories about parenthood and families, and describes the field of privacy by means of three typical stories: impossibility, opportunity and choice.

Key-words: parenthood, gays, lesbians, intimacy, heteronormativity.

\section{Parentalidades gays e lésbicas: trocas de narrativas}

\section{Resumo}

Neste artigo analiso histórias de gays e lésbicas em parentalidades e famílias. Meu objetivo é descrever o campo da privacidade a partir de três discursos típicos: o da impossibilidade, o da oportunidade e o da eleição. O estudo é baseado em uma pesquisa empírica e qualitativa, realizada entre os meses de setembro de 2008 e janeiro de 2009, em Buenos Aires, Argentina. Realizei 22 entrevistas com gays e lésbicas com filhos/as ou com projeto de tê-los.

Palavras-chave: parentalidade, gay, lésbico, privacidade, heteronormatividade.

Data de recebimento do artigo: 19/04/2012

Data de aprovação do artigo: 20/07/2012 